[Aus dem Institut für Infectionskrankheiten zu Berlin.]

\title{
Ueber die Dauer des Vorkommens von Choleravibrionen in den Dejecten von Cholerareconvalescenten. ${ }^{1}$ \\ Von
}

Dr. W. Kolle.

Durch die systematische Anwendung der Koch'schen Peptonmethode, ${ }^{2}$ in Verbindung .mit den schon seit langem benutzten Koch'schen Züchtungsmethoden, ist es bei der Untersuchung von Dejecten, Darminhalt, inficirten Gegenständen und Wasser dem bakteriologischen Choleraforscher möglich geworden, dem Choleravibrio bis in die entlegensten Schlupfwinkel nachzuspüren. So sind die specifischen Erreger der Cholera im Wasser, indem sie mittels des Gelatineplattenverfahrens nur von R. Koch ${ }^{3}$ in Indien, von C. Fränkel ${ }^{4}$ und von Lubarsch ${ }^{6}$ in Deutschland im Jahre 1892-je ein Mal gefunden waren, während der letzten Epidemieen in Deutschland fast überall da nachgewiesen worden, wo sie nach theoretischen Erwägungen erwartet werden durften. Die Constanz des Vorhandenseins des Koch'schen Vibrio bei der asiatischen Seuche, ist durch diese Methode in Tausenden von Fällen erwiesen. Auch bei den leichtesten Diarrhöen, die früher häufig als nicht specifisch betrachtet wurden, und als specifisch nicht sicher ${ }^{8}$ nachgewiesen werden konnten, hat man zur Zeit der Epidemieen, namentlich in Hamburg und Nietleben, ${ }^{7}$ den specifischen Keim gefunden und so ihre Natur anfgeklärt. Man hat ver-

${ }^{1}$ Eingegangen am 12. Juni 1894.

2 R. Koch, Ueber den augenblicklichen Stand der bakteriologischen Choleradiagnose. Diese Zeitschrift. Bd. XII.

3 Conferenz zur Erörterung der Cholerafrage. I. Jahrg.

4 Deutsche medicinische Wochenschrift. 1892. Nr. 41.

s Ebenda. 1892. Nr. 43.

${ }^{6}$ R. Griesinger.

7 R. Koch, Wasserfiltration und Cholera. Diese Zeitschrift. Bd. XIV. - Die Cholera in Deutschland während des Winters 1892/93. Ebenda. Bd. XV. 
mittelst dieser Methode den Koch'schen Kommabacillus an Wäsche und Gegenständen, die mit Entleerungen von Cholerakranken auch nur wenig beschmutzt waren, nachweisen können, man hat ihn ferner gefunden. bei scheinbar ganz gesunden Personen, welche in der Umgebung eines Cholerakranken sich aufhieiten, ohne die nöthigen Vorsichtsmassregeln zur Verhütung der Infection zu beobachten, oder welche der Infection in Folge Benutzung eines gemeinsamen, inficirten Brunnens oder dergl. ausgesetzt waren. Auch gelegentlich der Stettiner Cholera-Herbstepidemie $1893,^{1}$ die sich hauptsächlich in den Kreisen der Schiffer und der ärmeren Bevölkerung in der Umgegend des Hafens abspielte, ist es mir fast jedes Mal gelungen, bei mehr weniger zahlreichen Mitgliedern der betreffenden Familien, in denen ein Cholerafall vorgekommen war, Choleravibrionen nachzuweisen. $\mathrm{Zu}$ demselben Ergebnisse fübrten die in Bonn 1893 in der bakteriologischen Untersuchungsstation unter Leitung des Hrn. Prof. Dönitz ausgeführten systematischen Untersuchungen. Auch bei einem kleinen Choleraherd in Zerpenschleuse ob./Berlin haben die im Institut für Infectionskrankheiten vorgenommenen Untersuchungen gleiches Ergebniss gehabt. Sehr wichtig für die Würdigung dieser Thatsache ist die Beobachtung, dass bei zwei Mitgliedern einer Familie, in der ein Cholerafall vorgekommen war, bei der von mir mittels Peptonmethode vorgenommenen Untersuchung in den scheinbar normalen Dejectionen Choleravibrionen gefunden wurden, und dass mehrere Tage später diese Personen im Krankenhause auf der Evacuirtenstation, wo eine neue Infection ausgeschlossen war, an mittelschwerer bezw. leichter Cholera mit Diarrhöe erkrankten, in denen reichlich Cholerabakterien vorhanden waren.

Die grosse Bedeutung aller dieser Thatsachen für die Epidemiologie der Cholera ist von R. Koch in den Abhandlungen: „Wasserfiltration und Cholera" ${ }^{2}$ und "Die Cholera in Deutschland während des Winters 1892/93"3 eingehend auseinandergesetzt. Auf die grosse epidemiologische Bedeutung einer anderen Thatsache hat R. Koch dort, gestützt auf die Erfahrungen der Hamburger Nachepidemie, der Winterepidemie in Altona und Nietleben, sowie die gleich zu nennenden Angaben F. Fränkel's und Th. Rumpel's (Hamburg) gleichfalls schon hingewiesen, dass nämlich die Vibrionen nicht nur während des eigentlichen Choleraanfalles und in den diarrhöischen Stühlen sich finden, sondern noch eine grössere Zahl ron Tagen sich halten und durch das Züchtungsverfahren nach-

1 Die Beschreibung dieser Epidemie von R. Pfeiffer, welche in den Arbeiten aus dem Kaiserl. Gesundheitsamt erscheinen wird, ist noch im Druck.

2 Diese Zeitschrift. Bd. XIV.

-Ebenda. Bd. XV. 
weisbar sind. E. Fränkel und Th. Rumpel ${ }^{1}$ konnten nämlich die Cholerabakterien noch im Darminhalt eines am 18. Krankheitstage zur Section gekommenen Patienten nachweisen. Bei den weitbekannten Experimenten v. Pettenkofer's und Emmerich's ${ }^{2}$ wurden die specifischen Erreger 8 bezw. 11 Tage nach Aufnahme des Virus in den Excrementen wiedergefunden. Metschnik $\mathrm{ff}^{3}$ fand die Koch'schen Vibrionen in den Dejecten eines Arztes, der in Nachahmung' des Pettenkofer'schen Versuches den Cholerainfectionsstoff in Wasser aufgeschwemmt getrunken hatte und ziemlich schwer darnach erkrankt war, noch 19 Tage nach Ausführung des Experimentes.

Mit Rücksicht auf die Wichtigkeit dieser Beobachtungen habe ich im Auftrage des Hrn. Geheimrath Koch die Dejectionen von Cholerareconvalescenten in ausgedehntem Maasse untersucht.

Zuerst hatte ich im Juni vorigen Jahres Gelegenheit, den Verlauf einer Laboratoriumscholera genau bakteriologisch zu verfolgen; ich theile die Befunde im Einverständniss mit Hrn. Prof. R. Pfeiffer, den die Infection betraf, etwas ausführlicher mit, weil einige ganz bemerkenswerthe Beobachtungen dabei gemacht wurden. Hr. Prof. R. Pfeiffer hatte sich bei Anstellung von Thierexperimenten ${ }^{4}$ mit einer aus Hamburg bezogenen, dort frisch aus einem typischen Cholerafall gezüchteten Choleracultur inficirt und war an einem mittelschweren Choleraanfall mit profusen Diarrhöen erkrankt. Die am zweiten Krankheitstage vorgenommene bakteriologische Untersuchung des sehr dünnflüssigen Stuhles ergab die Diagnose Cholera durch das Gelatineplattenverfahren. Nach Einleitung einer diätetischen Therapie, sowie nach Verabreichung grosser Dosen von Calomel (in zwei Tagen $1 \cdot 6^{\mathrm{grm}}$ ) waren die Hxcremente fast steril. Sie enthielten nur einige Keime von Bact. coli in einer Oese. Cholerabakterien konnten selbst mittels des Peptonverfahrens nicht nachgewiesen werden, trotzdem eine ganze Anzahl Röhrchen mit dem Ausgangsmaterial beschickt waren. Nach Aussetzen der Calomeldarreichung stellten sich die Cholerabakterien aber in den Dejecten wieder ein und waren von da ab täglich nachzuweisen, Anfangs noch mittels des Gelatineplattenverfahrens, vom 20. Tage ab nur mittels des Peptonverfahrens, bis zum 33. Tage, rom ersten Krankeitstage gerechnet. Die Dajectionen waren Anfangs diarrhöisch, vom 15. Tage ab zuweilen von normaler Consistenz, erst vom 25. Tage dauernd normal. Das Blutserum von Prof. Pfeiffer zeigte, wie er selbst festgestellt

${ }^{1}$ Deutsche medicinische Wochenschrift. 1993. Nr.7.

2 Münchener medicinische Wochenschrift. 1892.

- Annales de l'Institut Pasteur. T. VII.

${ }^{4}$ Vgl. R. Pfeiffer, Studien zur Choleraätiologie und R.Pfeiffer u. Is saeff Ueber die specifische Bedeutung der Choleraimmunität. Diese Zeitschrift. Bd. XVII. 
hat, ${ }^{1}$ sechs Wochen nach der Erkrankung eine sehr ausgesprochene specifische schützende Wirkung gegen Intraperitonealcholera bei Meerschweinchen. Drei Monate später waren die specifischen Figenschaften des frisch entnommenen Blutserums nicht mehr nachweisbar.

Bei einer zweiten Laboratoriumscholerainfection, welche Hr. Prof. Pfuhl im September vorigen Jahres bei Anstellung von Thierversuchen acquirirte, waren die Cholerabakterien acht Tage lang in den Anfangs diarrhöischen, später consistenten Dejectionen nachweisbar. Die Erkrankung verlief hier so leicht, dass eine nennenswerthe Störung von Seiten des Allgemeinbefindens nicht eintrat, und die Krankheit nicht erkannt wäre, wenn nicht Prof. Pfuhl sie selbst bakteriologisch festgestellt hätte. Die bei dieser Erkrankung gewonnenen Culturen zeigten, Meerschweinchen intraperitoneal injicirt, die normalen Virulenzwerthe. Das Blutserum von Prof. Pfuhl zeigte nach den Versuchen des letzteren keine specifisch immunisirenden Eigenschaften gegen Cholera bei Meerschweinchen. ${ }^{2}$

Ein grosses Beobachtungsmaterial bot die schon genannte Stettiner Choleraepidemie im Herbst 1893. Ausser dem wissenschaftlichen Interesse, über die Zeitdauer des Vorhandenseins von Cholerainfectionsstoff und seine Menge im Stuhl der Cholerareconvalescenten etwas Näheres zu erfahren, lag hier noch die rein praktische, prophylaktisch gebotene Forderung vor, einen Menschen, bei dem Cholerabakterien gefunden waren, nicht eher von der Cholerastation oder aus der Beobachtung zu lassen, als bis seine Dejecte frei von Choleravibrionen waren. Die Excremente wurden als frei von Cholerabacillen dann angesehen, wenn die letzteren an drei auf einander folgenden Tagen nicht hatten gefunden werden können. Es muss ja zugegeben werden, dass die Gefahr einer Uebertragung der Seuche auf Gesunde von solchen Personen, welche relativ wenig Infectionserreger bei sich tragen und diese wenigen gewöhnlich in consistenten Excrementen entleeren, meistens nicht sehr gross ist. Aber da die Möglichkeit einer Uebertragung des Infectionsstoffes, wenn in Folge von Diätfehlern Eixacerbationen mit diarrhöischen Stühlen auftreten, nicht ausgeschlossen ist, so war die genannte Forderung um so berechtigter, als es sich um die Ausläufer einer Epidemie handelte, die leicht zu finden sind und unschädlich gemacht werden müssen, damit sie nicht den Ausgangspunkt

1 R. Pfeiffer, a. a. O.

${ }^{2}$ Diese beiden Laboratoriumsinfectionen beweisen, dass es auf eine grosse Menge des Infectionsstoffes, wie vielfach behauptet ist, zum Zustandekommen derInfection nicht ankommt. Denn da diese beiden geübten Bakteriologen mit allen Cautelen arbeiteten, so kann die Menge des Infectionsstoffes, welche die beiden Forscher aufgenommen haben, nur eine ganz minimale gewesen sein. 
für eine neue Epidemie an demselben oder einem anderen Orte bilden. In der Stettiner Epidemie hat denn auch niemals von Personen, die nach Ausfall der bakteriologischen Untersuchung als gesund, d. h. frei von Cholerabacillen bezeichnet und entlassen wurden, eine Ansteckung anderer Personen constatirt werden können.

Es wurden im Ganzen 50 Personen, welche die Cholera in den verschiedensten Formen, von leichtesten Diarrhöen bis zu schwersten asphyctischen Anfällen, durchgemacht hatten, während der Reconvalescenz untersucht. Die Resultate dieser Untersuchungen, ${ }^{1}$ bei denen ich von Hrn. Dr. Delius unterstützt wurde, sind auf der folgenden Tabelle zusammengestellt.

Tabelle.

\begin{tabular}{|c|c|c|c|c|c|}
\hline $\begin{array}{l}\text { 点 } \\
\text { 灾 }\end{array}$ & Narae des Patienten & $\begin{array}{l}\text { Klinische Form } \\
\text { der Krankheit }\end{array}$ & $\begin{array}{c}\text { Cholera- } \\
\text { vibrionen } \\
\text { zuerst } \\
\text { nachgewiesen }\end{array}$ & $\begin{array}{c}\text { Cholera. } \\
\text { vibrionen } \\
\text { zuletzt } \\
\text { pachgewiesen }\end{array}$ & $\begin{array}{c}\text { Folglich } \\
\text { Tage } \\
\text { vorhanden }\end{array}$ \\
\hline 1 & Bork. . . . & mittelschwer & $12 . / X$. & $15 . j X$. & 7 \\
\hline 2 & H. & sehr schwer & 12./X. & 18./X. & 7 \\
\hline 3 & M. . . . . . . & schwer & $12 . / \mathrm{X}$. & 18./X. & 7 \\
\hline 4 & Hahn . . . . & mittelschwer & 18./X. & $20 . x$. & 8 \\
\hline 5 & Loof. . & ” & 12./X. & $20 . / X$. & 9 \\
\hline 6 & R. . & leicht & 14. $/ \mathrm{X}$. & 18. $/ X$ & 5 \\
\hline 7 & Wollframm . . & mittelschwer & $8 . / \mathrm{X}$. & 18./X. & 11 \\
\hline 8 & Hedwig $\mathrm{K}$. & ganz leicht & $12 . / \dot{x}$. & 23. $/ X$. & 12 \\
\hline 9 & Frau Bühring . & schwer & $9 . / X$. & 19./X. & 11 \\
\hline 10 & Frau Warsow . & mittelschwer & $12 . / X$. & $19 . / X$. & 8 \\
\hline 11 & Frau Völker . . & " & 12. $/ \mathrm{X}$. & $29 . / X$. & 18 \\
\hline 12 & Frau Westphal . . & " & 8./X. & $23 . / X$. & 16 \\
\hline 13 & Oskar H. . . . & , & 13. $/ X$. & 23. IX. & 11 \\
\hline 14 & Paul H. . . . & leicht & $13 . / X$. & 23. $\mathrm{X}$. & 11 \\
\hline 15 & Fran Reglin ... . & schwer & 13./X. & 23./X. & 11 \\
\hline 16 & R. . . . . . & mittelschwer & 15./X. & 24. $/ \mathrm{X}$. & 10 \\
\hline 17 & Frau Trippensee & ganz leicht & 17./X. & $24 . / X$. & 8 \\
\hline 18 & Marie Trippensee & & 17. $\mathrm{X}$. & $24 . / X$. & 8 \\
\hline 19 & Breitag . . . . & leicht & $16 . / X$. & $25 . \mid x$. & 10 \\
\hline 20 & Bütow. & mittelscbwer & 19./X. & 26./X & 8 \\
\hline
\end{tabular}

1 Zur Herrichtang eines fliegenden Laboratoriums, in dem die sämmtlichen Cholerauntersuchungen vorgenommen werden, hatte mir Herr Dr. Schuchardt, Director des Neuen Stettiner Stadtkrankenhauses, ein Zimmer im Choleralazareth zur Verfügung gestellt. Seinem Entgegenkommen verdanke ich auch die reiche Ausbeute an Untersuchungsmaterial. 
(Fortsetzung.)

\begin{tabular}{|c|c|c|c|c|c|}
\hline $\begin{array}{l}\text { 究 } \\
\text { 声 }\end{array}$ & Name des Patienten & $\begin{array}{c}\text { Klinische Form } \\
\text { der Krankheit }\end{array}$ & $\begin{array}{c}\text { Cholera- } \\
\text { vibrionen } \\
\text { zuerst } \\
\text { nachgewiesen }\end{array}$ & $\begin{array}{c}\text { Cholera- } \\
\text { vibriunen } \\
\text { zuletzt } \\
\text { nachgewiesen }\end{array}$ & $\begin{array}{c}\text { Folglich } \\
\text { Tage } \\
\text { vorhanden }\end{array}$ \\
\hline 21 & Schwarzenb. . . & schwer & $16 . / x$. & 4./XI. & 20 \\
\hline 22 & . . . & leicht & $18 . / X$. & 4./XI. & 18 \\
\hline 23 & Hüp. . . & mittelschwer & 14. $X$. & $26 . \mid X$. & 13 \\
\hline 24 & Sauerbier & schwer & 4. $X$. & 26./X. & 22 \\
\hline 25 & Frau Stelter. . & sehr sehwer & 13. $/ \mathrm{X}$. & 26. $/ X$. & 14 \\
\hline 26 & Fran Siller . & leicht & 23./X. & $25 . / X$. & 3 \\
\hline 27 & Anna Siller . & , & $29 . / X$. & 4./XI. & 7 \\
\hline 28 & Gustav Siller. . . & , & $20 . / X$. & $26 . / X$. & 7 \\
\hline 29 & Arthur Westphal . & " & 12. $\mathrm{X}$. & $25 . / X$ & 13 \\
\hline 30 & Frau Sch. . . & , & $20 . / X$ & 2. XI. & 14 \\
\hline 31 & Emil Hüp. & ganz leicht & 19./X. & 29. X. & 11 \\
\hline 32 & Grete Bütow . . . & leicht & 19./X. & 30./X. & 12 \\
\hline 33 & Frau Engelbr. . . & mittelschwer & 23. $X$. & $30 . / X$. & 8 \\
\hline 34 & Max Braatz . . & , & 13. $/ X$. & $31 . / x$. & 19 \\
\hline 35 & Fran Karnitz . & sehr schwer & 16. X. & 31. $/ X$. & 16 \\
\hline 36 & Engelbr. & leicht & $20 . / X$ & 31./X. & 12 \\
\hline 37 & Franz Witte . . & " & 19. $/ \mathrm{X}$. & 2./XI. & 15 \\
\hline 38 & Helene Mohus . & ganz leicht & 29. X. & 4./XI. & 7 \\
\hline 39 & Dora Hüp. & leicht & 19./X. & 5. XI. & 18 \\
\hline 40 & Anna Arndt . . & , & $27 .[x$ & 7./XI. & 11 \\
\hline 41 & Hermann Arndt. & " & $28.1 X$. & 8./XI. & 11 \\
\hline 42 & Johannes Schröder . & ", & 26. X. & 8. $\mathrm{XI}$. & 14 \\
\hline 43 & Martha Schröder & $"$ & 26. $/ X$. & $10 . / X I$. & 16 \\
\hline 44 & Babenschneider. . & mittelschwer & 7. $/ x$. & 23./XI. & 48 \\
\hline 45 & Fră Hüp. . . . : & ", & 19. X. & $10 . / \mathrm{XI}$. & 23 \\
\hline 46 & Frau Bork. . . & leicht & 14. $X$ X. & 11.. XI. & 29 \\
\hline 47 & Frau Schröảer . & ” & 24. $/ \mathrm{X}$. & 8./XI. & 16 \\
\hline 48 & Gl. . & mittelsehwer & $20 . / x$. & 16./XI. & 28 \\
\hline 49 & Sehöfisch . . . & sehr schwer & 2./XI. & 20./XI. & 19 \\
\hline 50 & Marie Behnke . . & mittelschwer & 7./XI. & 20./XI. & 14 \\
\hline
\end{tabular}

Die täglich vorgenommene Untersuchung geschah mittels des Peptonverfahrens and ergab die Anwesenheit der Choleravibrionen fast stets. Nur in ganz vereinzelten Fällen kamen bei einzelnen Reconvalescenten Tage vor, an denen der Nachweis der Koch'schen Vibrionen nicht gelang. Mit der Schwere der Frkrankung scheint die Dauer des Vorhanden- 
seins ron Cholerabacillen in den Dejecten in keinem Verhältniss zu stehen, wie ein Blick in die Tabelle lehrt. Inwieweit das Verschwinden der Kommabacillen aus den Stühlen mit dem Eintritt oder einer Steigerung der Immunität des betreffenden Individuums in Zusammenhang steht, darüber konnten meine Untersuchungen nichts ergeben. Das Blutserum einiger Patienten zeigte, während sie noch entwickelungsfähige Choleravibrionen in ihren Dejecten hatten, stark immunisirende Eigenschaften gegen Intraperitonealcholera bei Meerschweinchen, während das anderer unter den gleichen Verhältnissen gar keine specifisch schützende Wirkung bei denselben Versuchsbedingungen erkennen liess. Auch über andere, z. B. locale Umstände, welche, ausser den als Immunität zusammenfassend bezeichneten Reactionen des Organismus, ein Verschwinden der Infectionserreger aus dem Darm bedingen könnten, ergaben die Untersuchungen nichts. Insbesondere liessen die zu verschiedenen Zeiten der Reconvalescenz isolirten Culturen Abweichungen in ihren Eigenschaften, namentlich in ihrer Virulenz gegenüber Meerschweinchen nicht erkennen von den typischen Culturen, die frisch bei schweren Cholerafällen gezüchtet waren, mit einer Ausnahme. Bei der am 40. Tage nach Beginn der Krankheit isolirten Cultur von Babenschneider (Fall 44) zeigte sich nämlich eine Herabsetzung der Virulenz; die dosis letalis minima einer 18stündigen Agarcultur war hier 1 Oese für ein Meerschweinchen von $300 \mathrm{grm}$ Gewicht.

Besonders sorgfältig konnten die Untersuchungen bei der oben beschriebenen Choleraerkrankung von Prof. Pfeiffer vorgenommen werden. Die während der Krankheit täglich isolirten Culturen zeigten sowohl unter einander, wie mit typischen Testculturen verglichen, constant dieselben morphologischen und biologischen Eigenschaften. Die Virulenz der am letzten Krankheitstage gezüchteten Cultur war genau dieselbe, wie diejenige der zu Beginn gewonnenen.

Sehr bemerkenswerth bezüglich der Zeitdauer,. innerhalb der, von dem Tage des ersten Auffindens der Cholerabakterien ab, diese letzten noch nachweisbar waren, sind die erhaltenen Resultate. Nur zwei Reconvalescenten beherbergten nachweisbar die Infectionserreger weniger als eine Woche bei sich, haben sie aber, wie man mit ziemlicher Sicherheit sagen kann, länger bei sich gehabt und entleert, da ihre Erkrankungen im Zeitpunkte der Untersuchung schon im Ablaufen begriffen waren. Bei sämmtlichen übrigen Fällen waren die Vibrionen mehrere Wochen nachweisbar, einmal (Nr. 44) sogar in den breiigen Excrementen, die am 48. Krankheitstage entleert waren. Ein fast ebenso lange dauerndes Vorkommen der Infectionserreger in menschlichen Dejectionen hat bisher nur Prof. Dönitz, als Leiter der bakteriologischen Untersuchungsstation 
zu Bonn im Herbst 1893, und zwar bei einer in Solingen an Cholera erkrankten Frau nachweisen können.

Hr. Prof. Dönitz hat die Liebenswürdigkeit gehabt, mir die über diesen Fall gemachten, gleich folgenden Notizen für diese Zusammenstellung zu überlassen.

Frau K., 71 Jahre alt, Papiermühle bei Solingen.

Am 9./IX. 93 wurden Choleravibrionen gefunden; zuletzt wurden sie am 25./X. nachgewiesen, also $46 \mathrm{Tage} n$ ach dem ersten Auffinden. Die alte Frau hat sich jedenfalls schon in den ersten Tagen des September inficirt, spätestens am 4. oder 5. September. Sie kam so von Kräften, dass der Tod Mitte October erwartet wurde. Dann erholte sie sich allmählich, während sie noch Choleravibrionen bei sich beherbergte. Von Mitte October an kamen zwischendurch Tage vor, wo Choleravibrionen nicht nachgewiesen werden konnten. Vom 25./X. 93 war sie dauernd frei von Cholerabacillen. Die Virulenzprüfung einer am 38. Tage der Erkrankung gewonnenen Cultur ergab, dass $1 \frac{1}{2}$ Oesen eine sicher tödtliche, 1 Oese eine zuweilen tödtende Dosis für Meerschweinchen von 300 bis $400 \mathrm{grm}$ Gewicht bildete. Jedenfalls war also die Virulenz etwas herabgesetzt.

Zum Schluss möchte ich besonders darauf hinweisen, wie die mitgetheilten Befunde von Neuem Beiträge zur Aufklärung und Richtigstellung von einigen bei der Epidemiologie der Cholera in Betracht kommenden Punkten liefern, welche von den Anhängern der Lehre von der autochthonen Entstehung der Cholera, sowie von denjenigen für die Beweiskraft ihrer Forschungsergebnisse benutzt werden, die ein Ueberwintern des Cholerakeimes ausserhalb des menschlichen Körpers annehmen. Wie schon R. Koch ${ }^{1}$ nachgewiesen hat, ist es unmöglich, in jedem einzelnen Falle den Faden von einem Choleraherde zu einem anderen aufzufinden. Dies wird um so einleuchtender, wenn man bedenkt, dass Menschen nach dem Ueberstehen des eigentlichen Choleraanfalles, während sie wie Gesunde ihren Geschäften nachgehen, ihren Wohnort verlassen u. s. w., noch 7 Wochen lang Infectionsstoff bei sich tragen und verbreiten können. Ein Mensch kann z. B. nach Ueberstehen eines Choleraanfalles in Indien von dort abreisen und nach vielen Tagen den Ausgangspunkt für eine Choleraepidemie auf dem Schiffe, auf dem er sich befindet, auf hoher See, oder in Europa werden, ohne dass man ihn nothwendiger Weise als Träger der Seuche erkennt. Dass ein Ueberwintern der Cholerabakterien hauptsächlich im Darm der Menschen in fortlaufenden Fällen, wie R. Koch ${ }^{2}$ es angenommen hat, stattfindet, wird durch meine Unter-

1 lib. cit.

2 lib. cit. 
50 W. Kolle: Choleratibrionen bei Cholerareconvalescenten.

suchungen bestätigt. Fälle, wie die unter 44, 46, 48 aufgeführten, sowie der Dönitz'sche Fall, erklären dies zur Genüge und zeigen, wie selten der Faden von einem Cholerafall, namentlich einem leichten, zum anderen selbst an demselben Orte, in demselben Hause, unter Umstânden zu finden seiu wird. Endlich zeigen die mitgetheilten Befunde, wie gross die Verbreitung des Infectionsstoffes ron einem einzigen Cholerakranken sein und wie sehr sie für viele Menschen gefahrvoll werden kann, wenn die infectiösen Dejectionen beispielsweise in öffentliche Wasserläufe gelangen. Sie liefern auch hier bestätigende Beiträge zu den Koch'schen Lehren. 